\title{
Paleoecología y gestión del combustible en la ocupación del auriñaciense arcaico de la cueva de El Castillo (Puente Viesgo, Cantabria)
}

\author{
Paloma Uzquiano* y Victoria Cabrera**
}

\section{RESUMEN}

El análisis antracológico correspondiente al nivel 18 (Auriñaciense arcaico) de la cueva del Castillo (Cantabria, Norte de España), ha puesto en evidencia una flora compuesta por Betula, Pinus $t$. sylvestris y Sorbus aria. Las dataciones ${ }^{14} \mathrm{C}$ en espectrometria de masa por acelerador obtenidas sobre carbón, sitúan este nivel en el Pleniglaciar medio (37-40Ka.). Las estimaciones paleoambientales obtenidas a través de los estudios de los glaciares de las montañas cantábricas y del Pirineo occidental, nos han permitido correlacionar la Antracología y el

Glaciarismo. El paisaje vegetal alrededor del habitat prehistórico estaría compuesto esencialmente por
ABSTRACT

Charcoal analysis from

archaeological levels of El Castillo (Cantabria, Northern Spain), have yielded Betula; Pinus $t$. sylvestris and Sorbus aria. The AMS ${ }^{14} \mathrm{C}$ obtained on the same samples place these levels on the Middle Pleniglacial (37-40Ka.). Palaeoenvironmental results obtained in glacial deposits from Cantabrian mountains and western Pyrenees let us to compare charcoal analysis and glaciation patterns. Landscape around this prehistoric settlement is formed essentially by pioneer vegetation cover along free-glace areas but under periglacial conditions. Altitudinal position of this site and

* Departamento de Estudios Clásicos. Facultad de Filosofía y Letras. Universidad de León. Campus de Vergazana, $s / n$. León. Correspondencia: Laboratorio de Arqueobotánica CEH-CSIC Duque de Medinaceli, 8. 28014 Madrid. E-mail: cehu118@ceh.csic.es.

** Departamento de Prehistoria y Arqueología. Universidad Nacional de Educación a Distancia. C/ Senda de Rey s/n 28040 Madrid. 
especies pioneras que han colonizado unos suelos libres de hielos pero sometidos a condiciones periglaciares debido a que este habitat estaba próximo de la cota altitudinal alcanzada por los glaciares de montaña. Estos serian responsables en parte del desfase altitudinal y zonal de la vegetacion si lo comparamos con las condiciones actuales. Los trayectos diarios recorridos por los grupos humanos a la búsqueda de recursos económicos diversos, entre ellos la leña para encender fuego, se verian influídos en gran parte por estos condicionantes ambientales.

PALABRAS CLAVE Paleoecología, Aprovechamiento de combustible, Paleolítico superior inicial, Glaciarismo, Región cantábrica. the glacial zones are similar. This particularity explain the altitudinal gap of the vegetation in this area. Diary routes taken by humans looking for their economical resources, as well as their woodfire, were influenced by these palaeoenvironmental conditions.

\section{KEY WORDS}

Palaeoecology, Woodfire management, Early upper Palaeolithic, Glacial deposits, Cantabrian region.

\section{EL YACIMIENTO DENTRO DE SU MARCO GEOGRÁFICO}

La cueva de El Castillo (fig. 1) está situada en el Valle del Pas (Cantabria). Desde un punto de vista topográfico, la posición horizontal de la Cordillera Cantábrica y la configuración hidrográfica (disposición NorteSur de los ríos principales), son los responsables de la fuerte compartimentación del territorio de Cantabria en una serie de Valles Transversales, cada uno de ellos con características propias (Cendrero et al., 1986), y que albergan un gran número de hábitats prehistóricos (Bernaldo de Quirós, 1982).

Esta cueva se abre con orientación N-NE en los afloramientos calcáreos de Monte Castillo (Puente Viesgo), a unos $30 \mathrm{Km}$ de distancia de la costa y a $195 \mathrm{~m}$ de altitud s.n.m. Estas elevaciones de altitud media $(400 \mathrm{~m})$, pertenecen a las últimas estribaciones de la Sierra del Escudo de Cabuérniga, primera barrera montañosa que atenúa las influencias del frente oceánico dominantes, y que separa a su vez, las zonas de «La Marina», con relieve y clima suaves, y de "La Montaña», donde el relieve es abrupto y el clima riguroso (Cendrero et al., 1986). La disposición horizontal de este relieve crea en ciertas áreas con buena exposición un 


\section{MAR CANTÁBRICO (GOLFO DE VIZCAYA)}

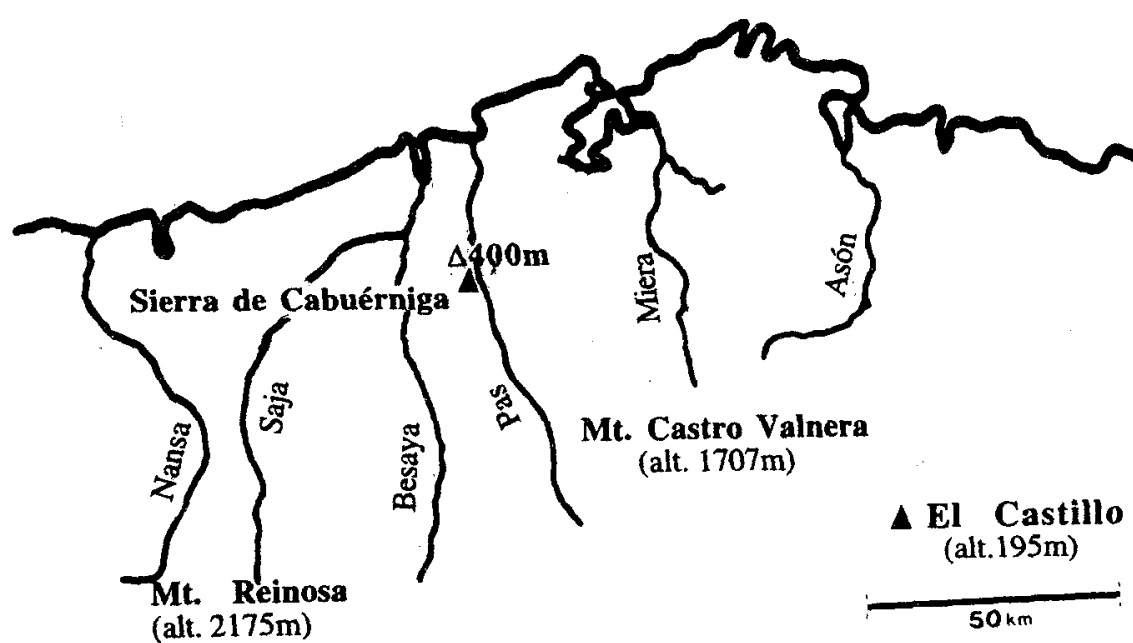

CORDILLERA CANTÁBRICA

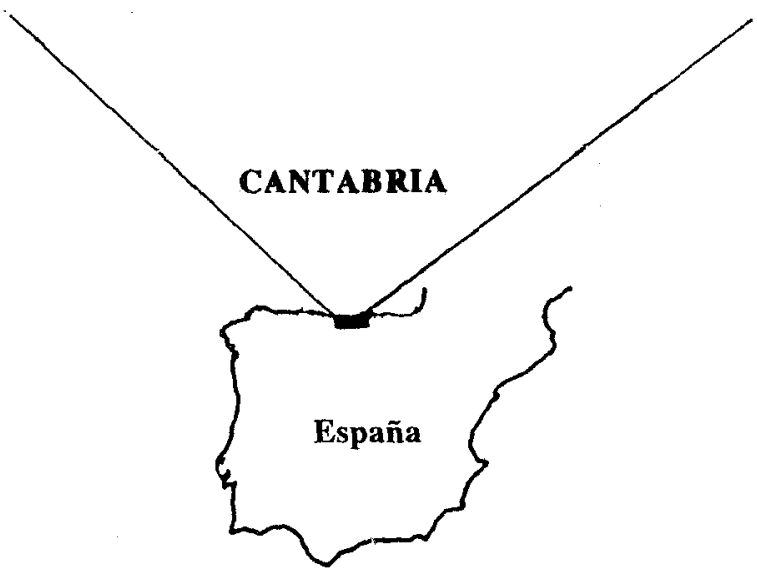

Fig. 1. Localización detallada de la cueva con todos los nombres de lugares mencionados en el texto (según Straus, 1992).

efecto de abrigo de los flujos dominantes del noreste, condicionando de este modo, al tipo de flora y de vegetación (Bertrand, 1974). La existencia de un gradiente altitudinal produce a su vez unos fuertes contrastes de vegetación en cortas distancias.

El Valle del Pas se encuentra en la actualidad fuertemente antropizado en sus tramos alto y medio por los Pasiegos. Estos pastores itine- 
rantes se desplazan con la familia y el ganado hacia el valle alto en verano y hacia el valle medio en invierno. El resultado de esta actividad es la existencia de praderías y zonas de pastos delimitadas con muros de piedra en todas las zonas bajas del valle. Árboles y arbustos han sido sistemáticamente eliminados de todo este área, quedando relegados hacia las partes más altas de las colinas donde la presión antrópica ha sido menor.

\section{VEGETACIÓN ACTUAL}

El territorio se encuentra dentro de la serie del robledal atlántico acidófilo con Quercus robur, Q. petraea y Castanea sativa (Dupont, 1962; Allorge, 1941; Cendrero et al., 1986). Esta formación ha desaparecido prácticamente en la actualidad no solo debido a la existencia de praderías y cultivos, sino tambien debido al efecto de las repoblaciones de Eucalyptus globulus. La extensión tan rápida de esta especie unido al poder antibacteriano de sus hojas que destruyen el horizonte de humus del suelo y la fuerte pluviometría de la zona, son los responsables de la fuerte degradación del suelo y de la extensión de especies de landa : Daboecia cantabrica, Erica tetralix, E. vagans, E. cinerea, Ulex europaeus, U. gallii (Duchaufour, 1947; Mayor y Díaz, 1977, Cendrero et al., 1986). Algunos ejemplares de Fagus sylvatica localizados hacia los $400 \mathrm{~m}$ de altitud en las cumbres de las montañas de los alrededores son el testimonio de la existencia de un hayedo que se extendía a más baja altitud (200 m) a principios del siglo $x x$.

El encinar cantábrico se encuentra bien desarrollado sobre substrato calcáreo en esta zona de Puente Viesgo. Quercus ilex, Arbutus unedo, Rhamnus alaternus, Phillyrea latifolia, Laurus nobilis, Ficus carica, etc. ocupan las zonas más abruptas de las partes bajas del valle donde la transformación del terreno en praderías ha sido imposible, dada la ausencia de suelo. Formaciones espinosas caducifolias constituídas por Prunus spinosa, $P$. amygdalus, Crataegus monogyna, Rubus ulmifolius, Lonicera periclymenum, Cornus sanguinea, Rosa sp., Ligustrum vulgare, Evonimus europaeus, Sambucus nigra, Tamus communis, se encuentran entremezcladas con las especies del encinar constituyendo un matorral arbustivo muy denso e impenetrable.

El fondo del valle ha sufrido grandes modificaciones. La extensión del pueblo con nuevas construcciones y casas de veraneo, los trabajos de remodelación del balneario y los cultivos de huerta intensivos han transformado las márgenes del río. Las especies mesófilas Corylus avellana y Fraxinus 
excelsior se disponen a lo largo del río al lado de algunos ejemplares de Alnus glutinosa, Populus alba, Salix spp., Hedera helix y los arbustos espinosos caducifolios mencionados anteriormente.

\section{MARCO CRONOESTRATIGRÁFICO Y CULTURAL}

La estratigrafía de esta cueva, conocida desde principios de siglo, ha ofrecido un larga secuencia de ocupaciones humanas que se han desarrollado a lo largo del Pleistoceno superior: Achelense, Musteriense, Auriñaciense inicial, Solutrense medio, Magdaleniense y Aziliense (Cabrera, 1984, 1989). La excavaciones que vienen efectuándose desde 1980 , se han centrado en el nivel 18 (fig. 2), subdividido en 18 b y 18c, y que corresponde al Auriñaciense inicial cantábrico (Cabrera Valdés et Bernaldo de Quirós, 1990; Bernaldo de Quirós and Cabrera Valdés, 1996; Cabrera et al., 1996).

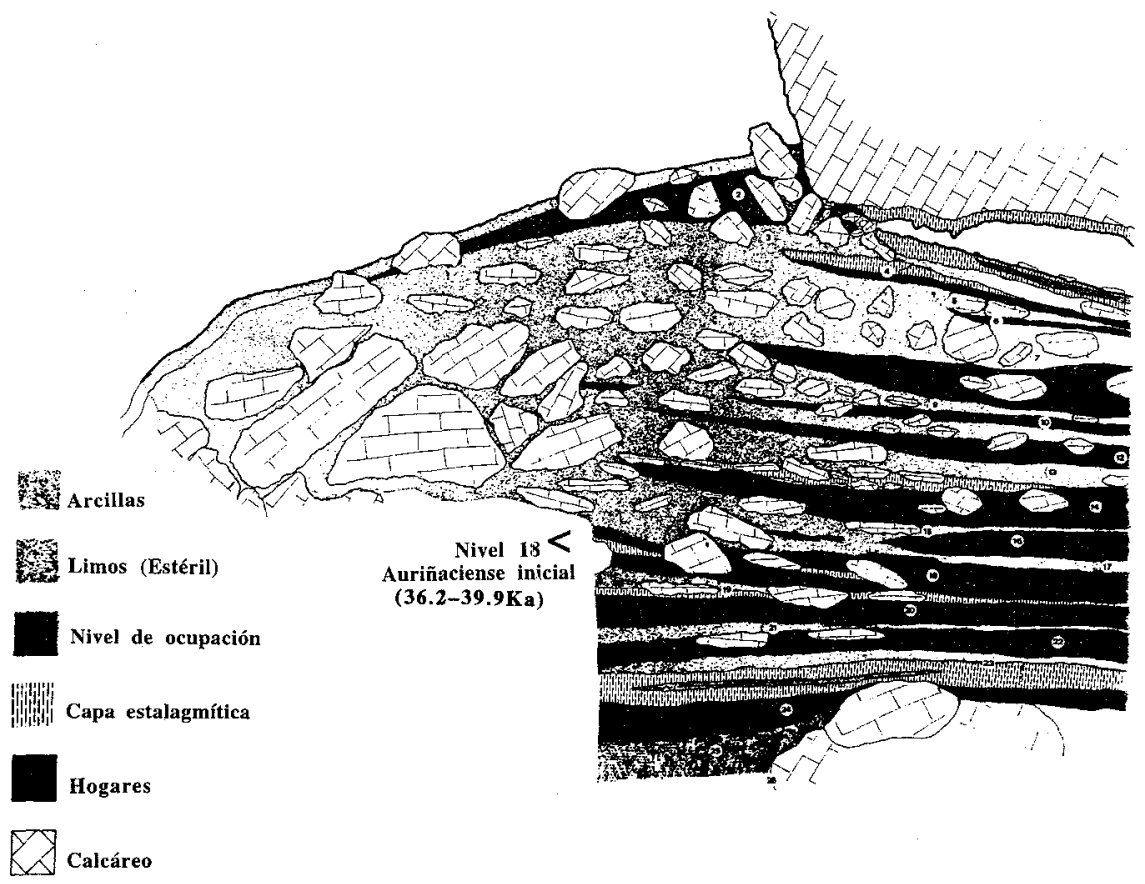

Fig. 2. Secuencia estratigráfica de El Castillo señalando la posición del nivel 18 objeto de estudio (según Cabrera, 1984) y las edades medias obtindas por las dataciones ESR

(Rink et al., 1996). 
En esta cueva se han efectuado diversas medidas radiométricas (Tabla 1). A partir de las fechas de $37.7 \pm 1.8 \mathrm{Ka}, 38.5 \pm 1.8 \mathrm{Ka}$ obtenidas por acelerador en $18 \mathrm{~b}$, y de $40 \pm 1.9 \mathrm{Ka}$ en $18 \mathrm{c}$, se estableció la media de $38.7 \pm 1.9 \mathrm{Ka}$ (Cabrera and Bischoff, 1989). Otras dataciones ${ }^{14} \mathrm{C}$ por acelerador fueron efectuadas en estas mismas capas estableciendo una media de 39.6 $\pm 1.5 \mathrm{Ka}$ (Hedges et al., 1994; Cabrera Valdés et al., 1996).

Tabla 1. Dataciones ${ }^{14} \mathrm{C}$ obtenidas por acelerador en el nivel 18 Auriñaciense inicial (según Cabrera Valdés et al., 1996)

\begin{tabular}{lllll}
\hline Referencia & Capa & Cuadro & \multicolumn{1}{c}{ Edad (BP) } & material \\
\hline AA-2406* $^{*}$ & $18 \mathrm{~b} 1$ & $\mathrm{~L}-15.4$ & $38500 \pm 1800$ & carbón \\
AA-2407 $^{*}$ & $18 \mathrm{~b} 2$ & $\mathrm{l}-14.6$ & $37700 \pm 1800$ & carbón \\
OxA-2473 $^{\circ}$ & $18 \mathrm{~b} 2$ & $\mathrm{H}-15.7$ & $37100 \pm 2200$ & carbón \\
OxA-2474 & $18 \mathrm{~b} 2$ & $\mathrm{H}-15.1$ & $38500 \pm 1300$ & carbón \\
OxA-2475 & $18 \mathrm{~b} 2$ & $\mathrm{~J}-14 \mathrm{a}$ & $40700 \pm 1600$ & carbón \\
OxA-2476 & $18 \mathrm{C}$ & $\mathrm{N}-17.3$ & $40700 \pm 1500$ & carbón \\
OxA-2476 & $18 \mathrm{C}$ & $\mathrm{N}-17.4$ & $41100 \pm 1700$ & carbón \\
AA-2405* & $18 \mathrm{C}$ & $\mathrm{N}-18.7$ & $40000 \pm 2100$ & carbón \\
OxA-2478 & $18 \mathrm{C}$ & $\mathrm{N}-17.6$ & $39800 \pm 1400$ & carbón \\
Gif-A89147 & $18 \mathrm{C}$ & $\mathrm{N}-17.3$ & $39500 \pm 2000$ & carbón \\
\hline
\end{tabular}

Edad media (*) : $38.7 \pm 1.9 \mathrm{Ka}$ (Cabrera and Bischoff, 1989).

Edaci media $\left({ }^{\circ}\right)$ : $39.6 \pm 1.5 \mathrm{Ka}$. (Hedges et al., 1994).

Las fechas ESR obtenidas recientemente (Rink et al., 1996) han dado una media para el Auriñaciense inicial de $36.2 \pm 4.1 \mathrm{Ka}$ que hace retroceder considerablemente la edad inicialmente establecida para la transición Paleolítico medio-superior en el sudoeste europeo (Cabrera and Bischoff, 1989; Bischoff et al., 1988, 1989). No obstante, la utilización de un nuevo programa de cálculo ha ofrecido una nueva media $(39.9 \pm 4.6 \mathrm{Ka})$ semejante a la obtenida por acelerador.

\section{MATERIAL Y MÉTODOS}

Se han analizado un total de 1441 fragmentos de carbón en este nivel de ocupación auriñaciense. Estos carbones se recuperaron de manera exhaustiva a partir de las técnicas de flotación manual y de cribado con agua (cribas de $\leq 5 \mathrm{~mm} y \leq 2 \mathrm{~mm}$ ) (Uzquiano, 1992a, 1997). Una vez seco el sedimento se procedía al triado de los diferentes materiales arqueológicos recogiendo los últimos restos de carbón que no habían po- 
dido recuperarse durante el proceso de flotación. Los carbones de mayor tamaño se han in situ, la mayoria de ellos han servido para las dataciones ${ }^{14} \mathrm{C}$ AMS mencionadas en el epígrafe anterior. Con esto queremos subrayar la contemporaneidad que tienen de las muestras analizadas tanto por la Antracología como por ${ }^{14} \mathrm{C}$ (Vernet et al., 1979; Vernet, 1992, 1995, 1997; Uzquiano, 1997). El estudio antracológico en extensión comprende una superficie de $43 \mathrm{~m}^{2}$. En toda esa superficie no se han detectado hogares claramente delimitados. A pesar de que la capa $18 \mathrm{c}$ presentaba una fuerte concentración de carbones, éstos han sido interpretados como una posible zona marginal de estructuras de combustión (o de vaciado de hogares), cuya localización estaría en el centro de la cueva, zona que fue enteramente vaciada de su sedimento durante las antíguas excavaciones (Obermaier, 1924; Cabrera, 1984). En la interpretación antracológica tenemos en cuenta como punto de partida a los grupos humanos y su economía donde la búsqueda diaria de combustible implica su selección dentro un territorio de explotación más o menos amplio. En este sentido, la distinción clásica entre carbones dispersos por el nivel de ocupación y concentrados en hogares que apuntan los trabajos de Antracología clásica (Chabal, 1991, Heinz, 1990, Figueiral, 1990), no ha sido tenida en cuenta ya que la selección de combustible está implícita en ambas categorías (Uzquiano, 1992a, 1997). Por otro lado, la ausencia de verdaderos hogares nos ha impedido la realización de un estudio en profundidad sobre la organización y acondicionamiento del hábitat en torno al fuego durante los inicios del Paleolítico superior cantábrico.

\section{RESULTADOS}

Betula pendula/B. pubescens son los táxones dominantes en el conjunto de las ocupaciones auriñacienses. Sorbus aria/Sorbus sp. son particularmente abundantes en la capa 18c. Pinus t. sylvestris solamente aparece de manera esporádica en $18 \mathrm{~b}$ y su representación es bastante discreta respecto a la de los abedules. La presencia de Leguminosas es igualmente débil (tabla 2). Los porcentajes de abedules están invertidos: el aumento que experiementan los abedules en 18b está seguido de un descenso brusco de los serbales (tabla 2; fig. 3), y coincide con la aparición esporádica del pino y un ligero aumento de las leguminosas. De este modo, el subnivel $18 \mathrm{~b}$ reflejaría una clara monoespecificidad frente al subnivel 18c. 
Tabla 2. Resultados antracológicos del nivel 18 (Auriñaciense arcaico) de la cueva de El Castillo (según Uzquiano, 1992a)

\begin{tabular}{lrrrr}
\hline \multicolumn{1}{c}{ NIVELES } & TAXA & \multicolumn{1}{c}{$18 C$} & \multicolumn{2}{c}{$18 B$} \\
& $N$ & $\%$ & \multicolumn{1}{c}{$N$} & \multicolumn{1}{c}{$\%$} \\
\hline Pinus t. sylvestris & & & 7 & 1,01 \\
Betula pendula & 297 & 41,19 & 219 & 17,14 \\
Betula pendula/pubescens & 210 & 29,12 & 379 & 52,63 \\
Sorbus aria & 128 & 17,75 & 22 & 3,05 \\
Sorbus sp. & 46 & 6,38 & 28 & 4,01 \\
Leguminosae & 3 & 0,41 & 9 & 1,25 \\
Indeterminables & 37 & 5,13 & 56 & 7,77 \\
\hline Total (1441) & 721 & & 720 \\
\hline
\end{tabular}

\begin{tabular}{ccc}
\hline$\%$ reagrupados & $18 C$ & $18 B$ \\
\hline Betula & 70,31 & 83,05 \\
Sorbus & 24,13 & 7,06 \\
\hline
\end{tabular}

$\%$

\section{EL CASTILLO: OCUPACIONES DEL AURIÑACIENSE ARCAICO}

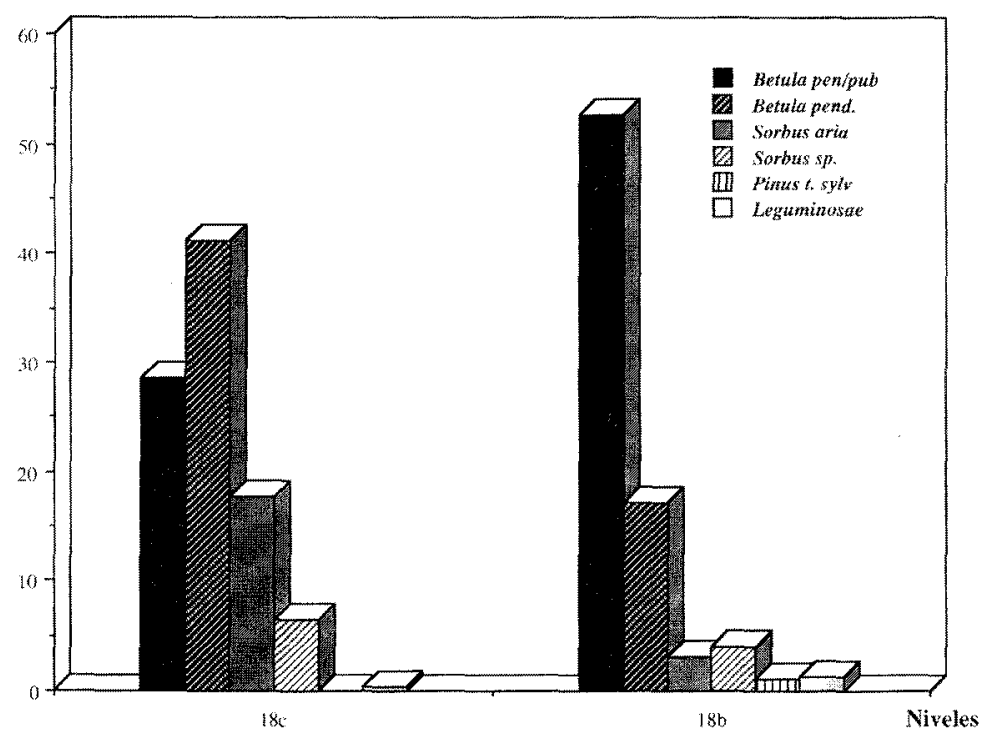

Fig. 3. Histograma con las fluctuaciones de las principales especies determinadas en el nivel Auriñaciense arcaico de El Castillo (según Uzquiano, 1992a). 
Una parte de las muestras analizadas en Castillo fueron determinadas como Betula verrucosa, siguiendo los criterios anatómicos de Jacquiot et al., (1973) y la nomenclatura propuesta en los atlas de anatomía al uso (Greguss, 1959, Schweingruber, 1978). Esta denominación fue cambiada por la de B. pendula, siguiendo la nomenclatura propuesta por Flora Europaea (Tutin et al., 1964). Otra parte de las muestras de Castillo fueron determinadas como $B$. pendula/pubescens (siempre siguiendo los criterios anatómicos de Jacquiot et al., 1973 y la nomenclatura de Flora Europaea), por presentar caracteres anatómicos ambíguos que nos hacían pensar, bien en una posible co-habitación de ambas especies, o bien en la posible existencia de ejemplares híbridos (Uzquiano, 1992a).

En lo que respecta a la nomenclatura, Flora Europaea distingue Betula pendula, Roth. y B. pubescens, Ehrh. (Tutin et al., 1964). Flora Ibérica, distingue dos grupos basándose en el aspecto de sus ramas (Castroviejo et al., 1990):

1. Ramitas del año y retoños pelosos con o sin glándulas resinosas $=$ Betula alba.

2. Ramitas del año y retoños glabros siempre con glándulas resinosas = Betula pendula.

El grupo denominado Betula pendula, Roth., engloba dos subespecies:

A. Betula pendula ssp. pendula donde quedan comprendidas las denominaciones de B. verrucosa, Ehrh. y de B. alba, L. (sensu Cadevall).

B. Betula pendula ssp. fontqueri.

El grupo denominado Betula alba, L., engloba a Betula pubescens, Ehrh., B. pubescens ssp. celtiberica, Rothm. y B. celtiberica, Rothm. (Castroviejo et al., 1990).

La nomenclatura Betula pubescens dada inicialmente para las muestras de El Castillo, fue cambiada por la de Betula alba (Uzquiano, 1992a) siguiendo los criterios de Flora Ibérica (Castroviejo et al., 1990), debido a la ubicación del yacimiento en la Península lbérica. Sin embargo la nomenclatura Betula alba de Flora lbérica se presta a confusión ya que en los dos grupos de abedules aparece esta denominación. Por este motivo, hemos preferido mantener los criterios de Flora Europaea Betula penduia y $B$. pubescens, atendiendo al esfuerzo realizado con la intención de distinguir ambas especies anatómicamente. Esta distinción es importante puesto que parte de la interpretación paleoecológica está apoyada en la posible co-existencia de estas dos especies de abedul en la zona, atendiendo a la repartición actual de ambas especies en el Cantábrico (Aseguinolaza et al., 1989; Mayor y Díaz, 1977). 


\section{DISCUSIÓN DE LOS RESULTADOS}

\section{Paleoetnobotánica y Paleoeconomía}

Estos resultados muestran una gran pobreza florística desde un punto de vista cualitativo. El elevado número de carbones analizado en estas dos capas no está en relación con el aumento progresivo de la riqueza específica ( . $^{\circ}$ de táxones). El tope máximo de riqueza específica se alcanza hacia los 200 fragmentos, siendo innecesario estudiar una cantidad mayor. Ello es debido a que los habitantes de la cueva en este período explotaron un medio caracterizado esencialmente por especies pioneras y colonizadoras de suelos libres de hielos: Betula y, en menor medida, Pinus. Este modo de explotación parece que se mantuvo a lo largo de toda la ocupación auriñaciense a juzgar por los altos porcentajes de abedul registrados en ambas capas.

Por otro lado, las fluctuaciones que Betula y Sorbus, registran en 18b y $18 \mathrm{c}$, así como la aparición de Pinus en 18b, nos permiten suponer tanto zonas de aprovisionamiento de combustible en el entorno del yacimiento diferentes, como una selección deliberada de especies distintas para quemar según fuese la funcionalidad de las distintas áreas de ocupación de la cueva a la que iban destinadas. Teniendo en cuenta el alto podeer calorífico del abedul, éste habría sido empleado en las partes más externas del hábitat y menos protegidas situadas hacia la entrada de la cueva, es decir, a lo largo de toda la superficie ocupada por el corte transversal y que corresponde a la capa 18b. Esto explicaría la monoespecificidad ya mencionada, el aumento que registra el abedul respecto a la capa precedente, $18 \mathrm{c}$, y la buena representación de las Leguminosas. Estas últimas especies son combustibles aptos para iniciar el proceso de combustión, siendo utilizados todavía con esta finalidad en algunas zonas de montaña del norte peninsular. A medida que penetramos hacia el interior de la cueva, Sorbus es particularmente abundante.

La capa 18c corresponde a otra zona de habitación más protegida situada en el corte longitudinal de la cueva. La presencia (18b)-ausencia (18c) del pino podría explicarse tambien por la existencia de un aprovisionamiento de leña en diferentes zonas (solanas y vertientes más al abrigo con suelos más secos, frente a umbrías y zonas de mayor humedad edáfica).

En resumen, la escasa diversidad ecológica nos permite suponer una utilización diferencial de la madera como combustible, pero la ausencia de verdaderos hogares nos impide saber de manera más precisa la funcionalidad de los mismos (culinaria, de iluminación, de acondicionamiento del hábitat o de trabajo o transformación de materias primas diversas, etc.). 
Paleoecología y gestión del combustible en la ocupación del auriñaciense arcaico...

La parte central del hábitat auriñaciense fue vaciada durante las excavaciones de principios de siglo. Hemos estudiado por tanto, zonas marginales del hábitat cuyo modo de vida en torno al fuego nos es desconocido, a pesar de que los diferentes excavadores (Alcalde del Río, Obermaier y Breuil), hayan señalado la existencia de hogares en sus respectivos cuadernos de excavaciones (Cabrera, 1984).

La avifauna estudiada en las excavaciones antíguas (Noval in Cabrera, 1984), señala una gran biodiversidad a través de la existencia de aves procedentes de biotopos diversos: bosque, pradera, escarpes rocosos, valles y zonas acuáticas, los cuales no han sido evidenciados por el antracoanálisis. Los grupos humanos en tanto que depredadores, realizaron numerosas expediciones alrededor del territorio que ocupaban estacionalmente a la búsqueda de diversos recursos económicos para cubrir sus necesidades cotidianas. Pensamos que la búsqueda de combustible también habría sido concebida según la naturaleza de los trayectos empleados y del tipo de recursos bucados en un medio de topografía diversa. Otros análisis antracológicos efectuados en cuevas paleolíticas del cantábrico con una cronología posterior a la época que nos ocupa, así lo han demostrado (Uzquiano, 1992a, 1992b, 1995, 1998). Esta particularidad no parece ser característica de las ocupaciones auriñacienses de esta cueva, atendiendo a los resultados obtenidos.

Por un lado hubiese sido necesario haber podido analizar todo el área central donde parece ser se encontraban los hogares con el fin de demostrar la existencia de una diversidad análoga a la percibida por la avifauna y poder correlacionar el modo de aprovisionamiento de combustible con la economía practicada. Por otro lado pensamos tambien que teniendo en cuenta la cronología, la escasa diversidad ecológica evidenciada guarda ciertas analogías con la paleoecología evidenciada por otros estudios efectuados en depósitos glaciolacustres y marinos de cronología similar. Teniendo en cuenta que el hábitat auriñaciense de Castillo es hasta la fecha el único que ha proporcionado información antracológica tan antigua, se hace necesario analizar muestras procedentes de otros hábitats prehistóricos más o menos contemporáneos de cara a conocer el combustible potencial disponible para los grupos humanos de los períodos más antiguos del Paleolítico.

\section{Paleoecología}

La abundancia de abedules puesta de manifiesto por el análsis de carbones, traduce condiciones propias del piso montano cantábrico actual. 
Betula pubescens aparece en la vertiente norte de la región cantábrica entre los 800 y $1.600 \mathrm{~m}$. de altitud entre formaciones caracterizadas por Quercus petraea, Sorbus aucuparia y S. aria (Mayor y Díaz, 1977). Su distribución geográfica revela una cierta afinidad a las condiciones húmedas propias del clima oceánico. Betula pendula es, por el contrario, menos abundante localizándose en algunos valles y montañas del interior de Asturias (Mayor y Díaz, 1977), así como en las sierras meridionales del País Vasco (Aseguinolaza et al., 1989). La distribución de este árbol es por tanto, más restringida, localizándose en las zonas donde la influencia oceánica de los flujos del noroeste disminuye por el efecto de la disposición de los relieves. Algunos ejemplares de abedul pueden encontrarse tambien entre las formaciones de hayedo en los claros de bosque dejados por las talas del haya. Con ello se pone de manifiesto la necesidad de luz que estas dos especies precisan, así como su carácter colonizador (Aseguinolaza et al., 1989).

Betula pubescens suele preferir los suelos húmedos mientras que Betula pendula suele encontrarse con frecuencia sobre suelos más secos pero tampoco desdeña los medios turbosos por ejemplo.

La abundancia de Betula pendula así como la posible co-habitación de las dos especies de abedul (Betula pendula/pubescens) en las ocupaciones auriñacienses de esta zona del valle medio del Pas, sugieren unas condiciones ambientales de transición hacia el clima continental, análogas a las que se observan actualmente en la vertiente sur de la Cordillera. La iiumedad que los abedules traducen no es forzosamente sinónima de abundancia de precipitaciones. La humedad pudo además estar condicionada por las características climáticas de este período (40Ka.): existencia de glaciares y proximidad de un Atlántico Norte sometido a bajas temperaturas de sus aguas de superficie.

La aparición tan esporádica del pino albar es difícil de interpretar. Por un lado, conocemos mal las perspectivas etnobotánicas derivadas del contexto arqueológico y las muestras polínicas analizadas han resultado estériles. Por otro lado, la vertiente norte de la Cordillera Cantábrica está actualmente desprovista de esta especie en estado espontáneo. En la vertiente sur se encuentran dos formaciones relictas cuyo carácter autóctono ha sido puesto en duda en ocasiones (Bertrand, 1974). Pinus sylvestris alcanza un gran desarrollo en la vertiente sur de los Pirineos, concretamente en la parte central alejada de las influencias marítimas (Dupias, 1985). Esta especie alcanza actualmente su optimum en el piso montano seco entre 1.000 y $2.000 \mathrm{~m}$. de altitud.

A la luz de estos resultados antracológicos, no podemos afirmar la existencia de formaciones de pino en el tramo medio del Pas, pero su 
presencia esporádica revelaría una procedencia localizada en ciertos sectores al abrigo de los vientos más húmedos teniendo en cuenta la disposición horizontal de la Sierra de Cabuérniga. La movilidad empleada por los grupos humanos en su búsqueda diaria de combustible así como de otros recursos económicos, habría puesto de manifiesto la existencia de estas zonas.

\section{Paleoclimatología}

El tipo de vegetación obtenida en Castillo podria ser característico de una de las fases interestadiales del Pleniglaciar medio puestas en evidencia en el norte de Europa (Behre, 1989; Behre and van der Plicht, 1992). Estos episodios de mayor o menor duración han sido objeto de controversias sobre todo en los depósitos polínicos de los yacimientos arqueológicos (Turner and Hannon, 1988) y muy especialmente en las cuevas cantábricas (Sánchez Goñi, 1993, 1994a, 1994b).

El registro de hielo de Groenlandia a vuelto a poner de manifiesto todas esas fases climáticas interestadiales (Dansgaard et al., 1993). La irregularidad que éstas han experimentado es debida a un comportamiento particular de la circulación del Atlántico Norte (Johnsen et al., 1992). Por otro lado, las oscilaciones tan rápidas de la temperatura de las aguas superficiales del mar han registrado a lo largo de los últimos 90Ka según los estudios de los últimos sondeos marinos efectuados en el Atlántico Norte (cores VM23-081, DSPD-609 y VM30-101k), coinciden a su vez con toda. esa serie de «eventos Dansgaard-Oeschger» mencionados (Bond et al,, 1993).

Si admitimos la existencia de estas oscilaciones climáticas en el Cantábrico, éstas no habrían sido sino variaciones en las condiciones de humedad. Teniendo en cuenta nuestros resultados (abundancia de abedules), la humedad en esta zona habría estado presente pero sujeta a fluctuaciones diversas que el registro marino ha detectado (Bond et al., 1993) y que en torno a $40 \mathrm{Ka}$., pudieron manifestar una tendencia hacia las condiciones de tipo continental mencionadas. La temperatura de las aguas del Cantábrico, así como la existencia de glaciares de montaña, serían responsables de estas oscilaciones y de la disminución de las condiciones de humedad.

Los datos sedimentológicos de la cueva han revelado no obstante, condiciones de tipo interestadial que han sido correlacionadas con el interstadio de Hengelo teniendo en cuenta la cronología (Cabrera Valdés et al., 1996). 


\section{Los glaciares y el sudoeste de Europa}

El desfase altitudinal de la vegetación que presenta el nivel del Paleolítico Superior Inicial de Castillo, se debe a la contemporaneidad de este hábitat con la existencia y proximidad de los glaciares. En el sur de Europa la situación es diferente en relación con el norte europeo. Los estudios llevados a cabo a nivel sedimentológico y palinológico en el piedemonte noroccidental de los Pirineos ya habían demostrado que el inicio de la deglaciación era anterior a 35Ka. (Jalut, 1990; Mardones et Jalut, 1982; Andrieu et al., 1988; Jalut et al., 1982, 1988). En los Pirineos centrales (Departamento de l'Ariège), estos estudios han detectado un retroceso del glaciar de l'Ariège hacia 20Ka. (Jalut et al., 1992). Las investigaciones llevadas a cabo en la vertiente sur (Cuenca del río Gállego), han demostrado tambien la existencia de una deglaciación precoz fechada en torno a $21 \mathrm{Ka}$. en los sedimentos de Bubal y Tramacastilla (Montserrat, 1992; Jalut et al., 1992). Otros estudios realizados sobre depósitos de erosión en los Pirineos aragoneses orientales (Serrano et Martínez de Pisón, 1994; Vilaplana, 1983) han trazado la evolución del glaciarismo Pleistoceno y Holoceno de la zona confirmando tambien, por paralelismo con la vertiente norte, una fase de retroceso glaciar análoga.

Mientras que el norte de Europa estaba sometido a condiciones pleniglaciares, situándose el período del máximo glaciar entre 20-18Ka., los glaciares pirenaicos ya habían comenzado su retroceso hacia $30 \mathrm{Ka}$., debido a la existencia de una mayor sequía (Jalut et al., 1992). La vegetación contemporánea del inicio de la deglaciación en Pirineos es muy fragmentaria porque los glaciares están presentes localmente. Los espectros polínicos son muy homogéneos y están compuestos principalmente por pólenes de herbáceas entre las cuales dominan Poaceae, Chenopodiaceae, y Artemisia. Los pólenes arbóreos están asimismo presentes siendo el pino el árbol dominante. Los paisajes de este período son sin duda abiertos con una cobertura vegetal discontínua (Jalut et al., 1988, 1992).

En las montañas cantábricas, las investigaciones del proceso de deglaciación están en sus inicios. Las zonas oriental y occidental de Cantabria han sido objeto de estudios diversos sobre glaciarismo.

En la zona oriental, los estudios de las morrenas de los ríos Miera, Asón y Gándara han puesto de manifiesto la existencia de una sola glaciación reciente, de edad pleistocena que ha destruído los vestigios de glaciaciones precedentes (Moñino et al., 1987). La cronología es incierta debido a la ausencia de datos cronológicos.

Los primeros estudios efectuados en la parte occidental de Cantabria pusieron en evidencia una glaciación contemporánea del Pleistoceno 
superior a partir de vestigios de morrenas localizados en las montañas de Reinosa a $2.175 \mathrm{~m}$ de altitud, en la parte noreste del Valle del Saja se localizaron a $1.200 \mathrm{~m}$, y en la parte noroeste del Nansa, a $1.500 \mathrm{~m}$ (Butzer, 1981). La cota altitudinal de los glaciares sería superior a los $1.000 \mathrm{~m}$ de altitud según este autor. Podríamos suponer que el desfase altitudinal de la vegetación auriñaciense de Castillo se debería entonces a una localización de glaciares a una altitud superior a la de los hábitats prehistóricos (Uzquiano, 1992a), originando con ello un «telescopage» de zonas de vegetación.

Estudios más recientes llevados a cabo por la Universidad de Cantabria han aportado nuevos datos concernientes a la altitud y a la extensión de estos glaciares. En las montañas pasiegas (Macizo de Castro Valnera, $1707 \mathrm{~m}$ de altitud), los estudios geomorfológicos han permitido detectar morrenas situadas a $450 \mathrm{~m}$ de altitud en el Valle del Asón y a $600 \mathrm{~m}$ de altitud en el Valle del Miera (Serrano, 1995). Si las cotas altitudinales en la zona de Puente Viesgo no exceden los $400 \mathrm{~m}$, algunos glaciares intramontanos más o menos próximos han descendido a una altitud análoga. En el Valle del Pas estos estudios no han permitido encontrar restos de morrenas si bien la morfología de la vertiente norte de Castro Valnera permite suponer que la lengua glaciar pudo alcanzar una altitud próxima a la evidenciada en el Valle del Asón (E. Serrano, com. pers.). Todos estos datos nos indican la existencia de glaciares situados a muy baja altitud cuya disposición era discontínua, lo que nos permite suponer que en los alrededores de Puenteviesgo existían zonas libres de hielo pero sometidas a condiciones de tipo periglaciar, dada la proximidad y la baja altitud alcanzada por los glaciares en este período. Este hecho explicaría el carácter poco desarrollado de la vegetación: un medio abierto caracterizado esencialmente por especies pioneras que han colonizado unos suelos libres de hielo, sometidos a condiciones de tipo periglaciar. El frío que supone la proximidad de estos glaciares y la cercanía del mar con unas temperaturas de aguas de superficie muy bajas, serían responsables del descenso de humedad anteriormente mencionado para esta zona. Las condiciones paleoambientales estarian muy próximas de las que actualmente existen en las montañas escandinavas (E. Serrano, com. pers.).

\section{CONCLUSIONES}

Los datos florísticos de la ocupación auriñaciense de El Castillo nos han permitido conocer desde un punto de vista antracológico las características del medio vegetal en el valle medio del Pas, hacia 40-38Ka. 
Desde un punto de vista cronológico la vegetación prehistórica de este período es aún poco conocida en el norte de España debido a que los resultados paleoflorísticos obtenidos en los depósitos arqueológicos han sido objeto de controversias (Sánchez Goñi, 1994b).

En lo que concierne a la Antracología, este trabajo constituye el primer estudio sistemático efectuado en el norte de España con una cronología tan antígua.

Desde un punto de vista paleoecológico la flora obtenida en ete yacimiento nos ha permitido poner en evidencia un doble desfase: altitudinal (formaciones próximas del piso montano entre 800 y $1.600 \mathrm{~m}$ de altitud) y zonal (condiciones de trasición hacia lo continental propias de las que actualmente existen en la vertiente meridional de la cordiliera).

En lo referente a la paleoclimatología, preferimos considerar que nuestros resultados antracológicos son más la consecuencia de una de las diversas fluctuaciones de humedad que esta región ha sufrido de manera irregular durante el Pleniglaciar, que el hecho de querer demostrar la existencia de interestadios análogos a los que tuvieron lugar en el norte de Europa. Esta humedad estaría atenuada por el frío procedente de la existencia de glaciares a baja altitud y por la proximidad de un mar sometido a unas temperaturas muy bajas de sus aguas de superficie, disminuyendo el aporte de humedad al continente. De ahí que las condiciones de tipo continental propias de la vertiente sur de la cordi!lera en la actualidad se localizasen en su vertiente norte en torno a $40 \mathrm{Ka}$.

Teniendo en cuenta la posición geográfica de la región cantábrica, nuestros resultados florísticos concuerdan tanto con la dinámica de la deglaciación propuesta para la vertiente norpirenaica como con las características de la vegetación arbórea (Jalut et al., 1992). Por otro lado, los recientes estudios de antíguas morrenas han revelado la existencia de glaciares intramontanos a muy baja altitud $(450-600 \mathrm{~m})$, sometiendo a las zonas libres de hielo a condiciones de tipo periglaciar. El escaso desarrollo de los suelos daría lugar a una cobertura vegetal discontínua, compuesta esencialmente por especies pioneras, colonizadoras, resistentes al frío y heliófilas. La vegetación leñosa puesta en evidencia en la ocupación auriñaciense de Castillo responde a estas mismas características. Sin embargo son necesarios nuevos estudios antracológicos de otros hábitats humanos contemporáneos del período glaciar en esta región para poder precisar mejor las carácterísticas del paisaje vegetal y el grado de aprovechamiento del medio en función del aprovisionamiento de combustible. 


\section{AGRADECIMIENTOS}

Estos resultados son parte de una tesis doctoral llevada a cabo por una de nosotras en la Université des Sciences et Techniques du Languedoc en Montpellier bajo la dirección del Profesor J.L. Vernet. A él le debo toda mi formación en Antracología.

Mi más sincera gratitud al Profesor G. Jalut de la Universidad Paul Sabatier en Toulouse por el interés y atención que siempre demostró en todos los trabajos antracológicos por mí realizados en la región cantábrica, y por todas esas discusiones inagotables que tanto sobre este manuscrito como en los anteriores han ido enriquecido considerablemente mi visión sobre la paleoecología cuaternaria del suroeste europeo.

Agradezco a E. Serrano, profesor titular de Geografía en la Universidad de Cantabria, sus críticas constructivas sobre un primer manuscrito, así como por haberme confiado algunos datos de los estudios más recientes sobre el glaciarismo en Cantabria.

\section{REFERENCIAS}

Allorge, P. (1941): Essai de synthèse phytosociologique sur le Pays Basque. Bulletin de la Societé Botanique de France t. $88: 291-356$.

AndRIEU, V.; HubSCHMAN, J.; JaLUT, G. \& HÉRAll, G. (1988): Chronologie de la déglaciation des Pyrénée françaises. Dynamique de sédimentation et contenu pollinique des paléolacs: application à l'interprétation du retrait glaciaire. Bull. ass. Française pour l'Etude du Quaternaire, 2-3:55-67.

aseguinolaza, C.; Gómez, D.; lizaur, X.; Montserrat, G.; Morante, G.; Salaverria, M.R.; Uribe-Echebarria, P.M. (1989): Vegetación de la Comunidad Autónoma del País Vasco. Ed. Gobierno Vasco. $361 \mathrm{p}$.

BEHRE, K.E. (1989): Biostratigraphy of the Last Glacial Period in Europe. Quaternary Science Reviews vol. $8: 25-44$. Pergamon Press.

BEHRE, K. E. \& VAN DER PLICHT, J. (1992): Towards an absolute chronology for the Last Glacial period in Europe: radiocarbon dates from Oerel, northern Germany. Vegetation History \& Archaeobotany, 1:111-117. Springer-Verlag.

BERNALDO DE Quirós, F. (1982): Los inicios del Paleolítico superior cantábrico. C.I.M.A. Monografía n. ${ }^{\circ} 8.343$ p. Santander.

Bernaldo de Quirós, F. and Cabrera Valdés, V. (1996): Raw material in the Palaeolithic of cueva del Castillo and in the Cantabrian region. In N. Moloney; L. Raposo; M. Santonja eds.: Non-flint stone tools and the Palaeolithic occupation of the Iberian Peninsula: 21-32. Tempus Reparatum. BAR International Series, 649.

BERTRAND, G. (1974): Essai sur la systématique du paysage: les montagnes cantabriques. Thèse de Doctorat. 3 vols. Université de Toulouse.

BischofF, J.L.; JULIA, R.; MORA, R. (1988): Uranium-series dating of the Mousterian occupation at Abri Romani, Spain. Nature, $332 ; 68-70$.

BISCHOFF, J.L.; SOLER, N.; MaRoto, J. \& JuliA, R. (1989): Abrupt Mousterian/Aurignacian boundary at c.40 ka BP : accelerator ${ }^{14} \mathrm{C}$ dates from l'Arbreda cave (Catalunya, Spain). Journal of Archaeological Science, $19: 49-62$.

Bond, G.; Broecker, W.; Johnsen, S.; McManus, J.; LaberRie, L.; Jouzel, J. \& Bonani, G. (1993): Correlations between climate records from North Atlantic sediments and Greenland ice. Nature vol. 365 : 143-147. 
BUTZER, K.W. (1981): Cave sediments, upper Pleistocene stratigraphy and Mousterian facies in Cantabrian Spain. Journal of Archaeological Science n. ${ }^{\circ} 8: 133-183$.

Cabrera Valdés, V. (1984): El yacimiento de la cueva de El Castillo. Biblioteca Praehistórica Hispana vol. XXII. $485 \mathrm{p}$.

- (1989): El Paleolítico medio cantábrico y sus relaciones con el área pirenaica. Espacio, Tiempo y Forma, S.I, Prehistoria y Arqueologia, t. 2 : 49-59.

CABRERA VALDÉs V. and BISCHOFF, (1989): Accelerator 14C dates for early upper Palaeolithic (Basl Aurignacian) at El Castillo cave (Spain). Journal of Archaeological Science, 19:577-584.

Cabrera Valdés, V. et Bernaldo de Quirós, F. (1990): Données sur la transition entre le Paléolithique moyen et le Paléolithique supérieur de la région cantabrique: revision critique. Paléolithique moyen récent et Paléolithique supérieur ancien en Europe. Colloque International de Némours, 9-11 Mai 1988. Mémoires du Musée Préhistorique de L'île-deFrance, 3: 185-188.

Cabrera Valdés, V.; Valladas, H.; Bernaldo de Quiros, F. et Hoyos, M. (1996): La transition Paléolithique moyen-Paléolithique supérieur à El Castillo (Cantabrie) : nouvelles datations par le carbone-14. C.R. Acad. Sci. París. t. 322, série lla: 1.093-1.098.

Cendrero, A.; Díaz de Terán, J.R.; Flor, E.; Francés, E. y Gonzálezz lastra, J.R. (1986): Guía de la Naturaleza de Cantabria. Ed. Librería Estudio. Santander. 350 p.

CHABAL, L. (1991): L'homme et l'évolution de la végétation méditerranéenne, des âges de metaux à la période romaine. Recherches anthracologiques théoriques appliquées principalement à des sites du Bas Languedoc. Thèse Physiologie et Biologie Des Organismes et Populations. U.S.T.L. Montpellier. 435 p.

Dansgaard, W.; Johnsen, S.J.; Clausen, H.B.; Dahl-Jensen, D.; Gundestrup, N.S.; Hammer, C.U.; HVIDBERG, C.S.; Steffensen, J.P.; SVEINBJörnsdotTiR, A.E.; Jouzel, J. \& BOND, G. (1993): Evidence for general instability of past climate from $250 \mathrm{kyr}$. ice core record. Nature, vol. $364: 218-220$.

Duchaufour, (1948): Recherches écologiques sur la chènaie atlantique française. Ann. Ec. Nat. Eaux et F. 332 p.

DupIAs, G. (1985): Végétation des Pyrénées. Carte de la végétation de la France $1: 200000^{\circ}$. Ed. CNRS. 209 p.

DuPONT, P. (1962): La flore atlantique Européenne: Introduction à l'étude du secteur lbéroAtlantique. Doc. Carte Prod. vég. Toulouse. $414 \mathrm{p}$.

FIGUFIRAL, I. (1990): Le nord-ouest de Portugal et les modifications de l'Ecosystème, du Bronze final à l'époque romaine, d'après l'anthracoanalyse de sites archéologiques. thèse Physiologie et biologie des Organismes et Populations. U.S.T.L. Montpellier $162 \mathrm{p}$.

Hedges, R.E.M.; Housley, R.A.; Bronk Ramsey, C. and van Klinken, G.J. (1994): Radiocarbon dates fron the Oxford AMS System : Archaeometry Datelist 18. Archaeometry, 36 (2) : 337 374.

HeINZ, C. (1990): Dynamique des végétations holocènes en Méditerranée Nord-Occidentale d'après l'anthracoanalyse de sites préhistoriques: Méthodologie et Paléoécologie. Paléobiologie Continentale vol. XVI (2) . U.S.T.L. Montpellier 212 p.

Hubschman, J. et JaLUT., G. (1989): Livre-Guide de l'excursion Glacier pyrénéen, versant nord/versant sud (Ossau-Gallego; Garonne-Noguera Ribagorçana). Toulouse 3-7 Mai 1989.

JaLuT, G. (1990): Le paléoenvironnement de la moitié occidentale des Pyrénées de 40000 BP à l'actuel: étapes de deglaciation et histoire de la végétation. In The Late Quaternary in the Western Pyrenean Region : 67-78. Universidad del Pais Vasco, Vitoria.

Jalut, G.; Delibrias, G.; Dargnac, J.; Mardones, M. \& Bonhours, M. (1982): A palaeoecological approach to the last 21000 years in the Pyrenees : the peat bog of Freychenede (alt. $1350 \mathrm{~m}$, Ariège, South France). Palaeogeography, Palaeoclimatology, Palaeoecology, 40: 321-359.

Jalut, G.; Andrieu, V.; Delibrias, G.; Fontugne, M.; Pages, P. (1988): Palaeoenvironment of the Valley of Ossau (Western French Pyrenees) during the last 27000 years. Pollen \& Spores $X X X, 3 / 4: 357-394$.

Jalut, G.; Montserrat Marti, J.; Fontugne, M.; Delibrias, G.; Vilaplana, J.M. and Julià, R. 1992. Glacial to Interglacial vegetation changes in the Northern and Southern Pyrenees: Deglaciation, vegetation cover and chronology. Quaternary Science Reviews, vol. 11: 449-480.

Johnsen, S.J.; Clausen, H.B.; Dansgaard, W.; Fuhrer, K.; Gundestrup, N.; Hammer, C.U.; IVERSEN, P.; JOUzel, J.; StaufFer, B. \& StefFENSEN, J.P. (1992): Irregular glacial interstadials recorded in a new Greenland ice core. Nature, vol. $359: 311-313$. 
Mayor, M. y Díaz, T.E. (1977): La flora asturiana. Col. Popular Asturiana n. 27. Ayalga Ed. Oviedo. $1.047 \mathrm{p}$.

MONTSERRAT, J. (1992): Evolución glaciar y postglaciar del clima y la vegetación en la vertiente sur del Pirineo: estudio palinológico. Monografías del Instituto Pirenaico de Ecología $n .^{\circ} 6$, CSIC, Zaragoza. $147 \mathrm{p}$.

Moñino, M.; CendRero, A. y Díz de TeRÁn, J.R. (1988): dinámica glaciar cuaternaria en la vertiente norte de Castro Valnera, Cantabria. // Congreso Geológico de España vol. 1: 398-402. Granada.

Obermaler, H. (1924): Fossil man in Spain. New Haven CT. Yale University Press.

Rink, W.J.; Schwarcz, H.P.; LeE, H.K.; Cabrera, V.; Bernaido de Quirós, F.; Hoyos, M. (1996): ESR Dating of Tooth Enamel : comparison with AMS ${ }^{14} \mathrm{C}$ at El Castillo cave, Spain. Journal of Archaeological Science, 23 : 945-951.

SÁNCHEZ GoNil, M.F. (1993): De la taphonomie pollinique à la reconstitution de l'environnement. L'exemple de la region cantabrique. British Archaeological Reports. International Series, 5586. Oxford. $207 \mathrm{p}$.

- 1994a. The identification of European upper palaeolithic Interstadials from cave sequences. AASP Contribution series $n .^{\circ} 29: 161-182$.

- 1994b. L'environnement de l'homme préhistorique dans la region cantabrique d'après la taphonomie pollinique des grottes. L'Anthropologie, $t .98 n .^{\circ} 2-3: 379-417$.

SerRANO, E. (1995): Geomorfología glaciar del Alto Trueba (Burgos). In G. Meaza; J.C. García Codrón; J. Arnáez eds. Libro-Guía de las excursiones de campo de las XI Jornadas de Campo de Geografía Física : 91-102. 22-25 Mayo 1995.

Serrano E. y Martínez de PISÓn, E. (1994): Geomorfología y evolución glaciar en el Pirineo aragonés oriental. In C. Marti Bono \& J.M. García Ruiz eds.: El glaciarismo surpirenaico: nuevas aportaciones : 33-64. Geoforma ediciones, Logroño.

Straus, L.G. (1992): Iberia before iberians. The Stone age Prehistory of Cantabrian Spain. University of New Mexico Press. $336 \mathrm{p}$.

Uzquiano, P. (1992a): Recherches anthracologiques dans le secteur Pyréneo-cantabrique (Pays Basque, Cantabria et Asturias): Environnements et relations homme-milieu au Pléistocène supérieur et debut de l'Holocène. Thèse Physiologie et Biologie des Organismes et Populations. U.S.T.L. Montpellier. 400 p.

- (1992b): L'homme et le bois au Paléolithique en région cantabrique, Espagne. Les exemples d'Altamira et El Buxu. En J.L. Vernet éd. : Les charbons de bois, les anciens ecosystèmes et le rôle de l'homme. Bull. Soc. Bot. Fr., 139. Actualités bot., 2/3/4 : 361-372.

- (1995): La disparition de Picea à la fin du Pléistocène supérieur en region cantabrique d'après l'anthracoanalyse: déterminisme climatique et anthropique. Comptes rendus Acad. Sci. París. t. 321 série lla : 545-551.

- (1997): Antracología y métodos. Implicaciones en la Economía prehistórica, Etnoarqueología y Paleoecología. Trabajos de Prehistoria, vol. 54 (1) : 145-154. C.E.H./CSIC, Madrid.

- (1998): La végétation cantabrique de 13000 à 9000 BP d'après l'analyse anthracologique. Habitats et ramassages de bois dans un milieu changeant. Actas del XIII Congreso U.I.S.P.P. Forli (ltaly). Vol. I : 477-482.

VERNET, J.L. (1991): L'histoire du paysage humanisé révèlée par les bois carbonisés. In J. Guilaine éd. : Pour une archéologie agraire: 369-408. Armand Colin, París.

- (1992): Charbons de bois et Paléoenvironnement méditerranéen. In D. Marguerie et J.Y. Hunot éds.: Les bois archéologiques. A.G.O.R.A., $2: 2\}-25$. Université de Rennes I.

- (1995): Anthracologie, Biostratigraphie et Rélations Homme-Milieu en région méditerranéenne. En L'Homme et la dégradation de l'Environnement. 175-184. Actes des XVè Rencontres Internationales d'Archéologie et d'Histoire d'Antibes. Juan les-Pins. Ed. APDCA. Sophia-Antipolis.

- (1997): L'homme et la forêt méditerranéenne de la Préhistoire à nos jours. Collection des Hespérides. Ed. Errance, París. 248 p.

VERNET, J.L.; BAZILE-ROBERT, E.; EVIN, J. (1979): Coordinations des analyses anthracologiques et des datations asbsolues sur les charbons de bois. Bulletin de la Societé Préhistorique Française t. 76 n. $3: 76-79$.

VILAPLANA, J.M. (1983): Quaternary Glacial Geology of Alta Ribagorça Basin (Central Southern Pyrenees). Acta Geológica Hispánica. t. 18 (3/4) : 217-233. 